\title{
INFLUENCIA DE FACTORES INTERNOS Y EXTERNOS SOBRE EL RENDIMIENTO ESCOLAR EN LA EDUCACIÓN PRIMARIA BAJO UNA PERSPECTIVA GLOBAL
}

\author{
Influence of internal and external factors on the school performance in \\ primary education under a global perspective
}

\section{Carlos Antonio Del Castillo Guardamino $^{7}$}

\section{RESUMEN}

Objetivo: Esta investigación examina la relación de diversos factores sobre el rendimiento escolar, medido a través de la prueba PISA, que se aplica en más de 60 países cada 3 años. El objetivo es analizar cuáles de ellas tienen más relación en los resultados.

Método: Su orientación principal es la evaluación de la capacidad de comprensión lectora y matemática en varios niveles. Existen muchas variables que pueden influir en dichos resultados, clasificadas genéricamente como internas (inherentes al sistema educativo) y externas (del entorno o ambiente).

Resultados: Los datos utilizados provienen del Banco Mundial de los últimos 10 años (últimos datos del 2012). Los resultados indican que si bien cada una de las variables seleccionadas ejerce alguna influencia sobre la calidad educativa medida a través del rendimiento escolar, son las variables externas las que tienen un mayor poder explicativo sobre los resultados, lo cual corrobora los hallazgos de muchos estudios previos realizados a nivel de países y regiones.

\footnotetext{
${ }^{7}$ Magister en Investigación en Ciencias de la Administración. Profesor de la Universidad ESAN.
}

Conclusión: Esta investigación ha simplificado a nivel global un modelo que buscaba probar que, las premisas que se generaron hace varias décadas sobre la importancia de factores no directamente ligados al sistema educativo, aún siguen vigentes, y que son aquellos elementos del entorno, de las condiciones macroeconómicas y de bienestar, los que ejercen una mayor influencia sobre los resultados de las pruebas de rendimiento escolar.

\section{PALABRAS CLAVES}

Rendimiento escolar, calidad educativa, comprensión

\section{ABSTRACT}

Objective: This research examines the relationship of various factors on student achievement, as measured by the PISA test, which is applied in more than 60 countries every three years. The aim is to analyze which of them have more to results.

Method: Its main focus is the evaluation of the ability of reading and mathematical understanding at various levels. There are many variables that can influence those outcomes, generically classified as internal (inherent in the education system) and external (environmental or ambient).

Results: The data used come from the World Bank for the past 10 years (most recent data 2012). The results indicate that although each of the selected variables exerts some influence on the quality of education 
as measured by the academic performance are external variables that have greater explanatory power of the results, which corroborates the findings of many previous studies made at the level of countries and regions.

Conclusion: This investigation has simplified global model that sought to prove that the premises that were built decades ago on the importance of factors not directly linked to the education system, still in effect, and which are those elements of the environment, the macroeconomic and welfare conditions, which exert a greater influence on the results of achievement tests.

\section{KEY WORDS}

School performance, educational quality, understanding

\section{INTRODUCCIÓN}

La medición de la calidad educativa es uno de los temas más importantes de la agenda educativa mundial. Los responsables de la educación en todos los países han tomado conciencia de la necesidad de evaluar los sistemas educativos y los niveles de aprendizaje de los estudiantes (Carlson, 2000).

En base a los resultados de las pruebas PISA (Programme for International Student Assessment, se basa en el análisis del rendimiento de estudiantes a partir de unos exámenes mundiales que se realizan cada tres años y que tienen como fin la valoración internacional de los alumnos), se califica al rendimiento escolar en el Perú como deficiente, pues lejos de converger hacia niveles internacionales y a pesar del incremento del gasto público en educación, se ubica en un lugar poco privilegiado.

Si bien existen pocas estadísticas del rendimiento escolar en América Latina, las existentes tienden a señalar que el rendimiento escolar en América Latina y el Caribe es significativamente menor al del mundo desarrollado y al de los países asiáticos. Se han hecho innumerables esfuerzos por incidir directamente en los factores directamente ligados al sistema educativo (horas de clase, capacitación a docentes, remuneraciones y prestaciones a docentes, infraestructura educativa), pero a nivel de educación pública sobre todo, los resultados de las evaluaciones del rendimiento escolar no han mostrado progresos significativos (Agüero \& Cueto, 2004).

Resulta importante en este escenario, identificar políticas para revertir esta tendencia. Nos interesa por tanto analizar la influencia de los factores externos, en especial los orientados a la economía, las condiciones de bienestar social y la infraestructura de soporte a la educación, de tal forma que puedan ser incorporados como parte de la estrategia integral de mejora de la educación escolar en el Perú.

Diversos estudios y trabajos han sido realizados para analizar los factores que afectan el rendimiento escolar. Gran parte de ellos se centró en tomar en cuenta dos tipos de causas: las relacionadas con el sistema educativo (llamadas internas), y aquellas que se orientan al entorno o contexto económico-social (llamadas externas) (Mella \& Ortiz, 1999). 
En 1964, luego de la aprobación de la Ley de Derechos Civiles en los Estados Unidos, se realizó una encuesta nacional para conocer si todos los alumnos (independientemente de su condición económica, raza, religión), tenían las mismas oportunidades educativas, encargando este trabajo financiado con fondos federales a James S. Coleman. El informe oficial se tituló "Equality of Educational Opportunity Study", aunque se le conoce en la actualidad como Informe Coleman. La conclusión del estudio fue que los insumos o factores escolares tenían un efecto poco significativo sobre el desempeño escolar, siendo por el contrario los factores socioeconómicos o del entorno los que ejercian mayor influencia (Coleman et al., 1966)

Otro estudio, realizado en Gran Bretaña (Jencks, 1971), no solo confirmó años después los hallazgos de Coleman (es decir que el impacto de la escuela sobre los resultados era muy limitado), sino que probó que, en un sistema educativo con una estructura totalmente diferente a la norteamericana, seguían siendo los factores del entorno los más importantes.

Creemers (1992), en otro estudio similar, señaló que el estatus socioeconómico es la variable más importante en la explicación de los puntajes de logro escolar, aunque no descartó que las variables orientadas al sistema educativo también contribuyen pero en menor medida. Otro estudio realizado de manera más amplia para los países europeos (Mortimore, 1988) señalaba que la variable tiempo de instrucción no contribuye mucho a la explicación de la efectividad escolar.

Estudios realizados para la realidad latinoamericana como el realizado por Himmel (1984), exploró la influencia de un amplio conjunto de variables sobre el rendimiento escolar, llegando una vez más a la conclusión que el nivel socioeconómico es la variable más importante en la explicación del rendimiento escolar.

Un estudio bastante conocido y que fue realizado para Brasil (Lockheed \& Bruns, 1990) por encargo del Banco Mundial, mostró que la diferencia en el rendimiento de los alumnos (manifestada por el éxito en las pruebas previas de selección para ingresar a la universidad) se atribuía al tipo de escuela, algo que de alguna manera se orientaba a la situación socioeconómica y al entorno de los estudiantes.

La CEPAL, en una investigación orientada a la instrucción primaria (Espinola \& Martínez, 1996) buscó identificar que variables influyen más en el logro de la enseñanza a ese nivel básico, siendo el bienestar de la familia atribuible a su posición económica el que tiene importancia gravitacional para explicar el resultado escolar. $Y$ al igual que los estudios anteriores, concluye que si bien la influencia de los factores internos o del sistema educativo no es depreciable, no llegan a explicar contundentemente el rendimiento escolar como si lo hacen los factores externos o del entorno (Aitken \& Longford, 1986).

Otros estudios llegan incluso a conclusiones más específicas. Fitz y Gibbon (1992) señalaban que dentro de las variables internas, los alumnos son más dependientes de la escuela en el área de comprensión matemática que en lectura. Adicionalmente señalan que los alumnos de menores niveles socioeconómicos dependen "en mayor medida" del sistema escolar, por tanto 
son más vulnerables a la buena o mala influencia de la escuela. Se señaló incluso que un factor sensible como es el tamaño de la clase (número de alumnos por aula o ratio alumnos por maestro) es un factor que tendería a condicionar mejores resultados. Sin embargo, un año después las investigaciones de Heyneman y Loley (1983) señalaron que no existe evidencia específica de este asunto.

La identificación de condiciones socioeconómicas y del entorno que intervienen en los desempeños y aprendizajes escolares, implica indagar en la capacidad de las familias para apoyar con recursos materiales y culturales (Saavedra et al., 1997). Por tanto, la mayoría de investigaciones concluye que a una mayor disponibilidad de ingresos (condiciones económicas, bienestar, soporte en servicios, etc.) se puede impactar decisivamente en el resultado escolar, porque implicaría una capacidad mayor de pago (orientando la matrícula a escuelas privadas), una mejor infraestructura, mejor alimentación, mejor salud, mejor transporte a la escuela, mejores condiciones en general para el estudio.

Para comprender el efecto de estas interacciones sobre el rendimiento escolar, es necesario entender cuáles son las variables del entorno. Un caso particular es lo que se conoce como interacciones sociales, cuyos efectos se dividen en tres (Mella \& Ortiz, 1999). El primero, denominado efecto de contexto, cuando el desempeño de un alumno se ve afectado por las características de entorno cercano. El segundo, llamado endógeno, implica que el rendimiento de un alumno depende del rendimiento promedio de sus compañeros, independientemente de las características de estos. El tercero, conocido como efecto correlacionado, supone que los alumnos de un determinado sistema educativo se comportan similarmente porque son educados bajo condiciones similares.

La mayoría de estimaciones basadas en una función que relacione el rendimiento escolar con variables tanto educacionales como no educacionales, reafirman la importancia que tienen las variables del hogar en el desempeño escolar de los niños, hipótesis que ha sido confirmada en numerosos estudios internacionales. En el caso de Bolivia, no sólo la educación de los padres es un determinante importante del resultado estudiantil, sino que también otras variables que reflejan más particularmente el contexto de un país en desarrollo, como las desventajas que enfrenta la población de origen indígena, los niños que por razones de estudio o económicas no viven con sus padres y el hecho que la madre ayude en las tareas escolares a los niños (Mizala et al., 1999).

Sin embargo, lo particular de este estudio es que demuestra que las variables del colegio y del profesor son también importantes. Variables como la experiencia del profesor, que el profesor deje tareas todos los días, indicadores de infraestructura y de tamaño del colegio son estadísticamente significativos.

En términos generales los colegios públicos presentan un peor desempeño escolar que los establecimientos privados. Ello plantea un fuerte desafío para la política educacional de los países en desarrollo, dado que será necesario disminuir estas brechas de rendimiento escolar para que la educación efectivamente se transforme en un vehículo de movilidad social y de mejoramiento de la distribución del ingreso (Saavedra et al., 1997). 
Existe aún mucha controversia sobre el efecto específico de otros factores, que si bien están orientados a la mejora de la calidad educativa, no han mostrado un real impacto desde acciones de política: gasto en remuneraciones y prestaciones en maestros, disminución de la deserción, incremento del número de maestros, etc. Cabe resaltar que un estudio hecho en el Perú revela que las familias asumen aproximadamente la tercera parte del financiamiento total de la escuela pública (Saavedra et al., 1997), lo que implica que el efecto del gasto público en educación escolar tiene una importante contraparte económica desde las familias.

\section{MATERIALES Y MÉTODOS}

Dado que no existen datos para todos los años ni para todos los países sobre la prueba PISA y las variables a considerar como independientes, se consideró conveniente para el modelo calcular los promedios de algunos indicadores para el periodo analizado. Esto permitía dos ventajas: permitía evaluar transversalmente las variables en un período de tiempo cronológico. Pero adicionalmente permitía minimizar el efecto de falta de algunos datos en algunos años. Esto es importante porque lamentablemente no existen estadísticas para todas las variables y para todos los años de la muestra tomada. Como se mencionó, las pruebas PISA no se toman todos los años, pero sin embargo los factores que la afectan según el modelo planteado no necesariamente corresponden al año en que se toma la medición, sino que se asume que son los factores o variables de años anteriores las que afectan los resultados del rendimiento escolar.

Para facilitar el análisis, y dado que los resultados se orientan principalmente a evaluar el rendimiento a nivel de primaria, en algunos casos solo se ha tomado los datos que corresponden a ese nivel, y en otros el total de niveles y grados (cuando ha sido imposible desagregar).

Adicionalmente se tenían dos resultados correspondientes a las mediciones de calidad educativa a través del rendimiento en las pruebas PISA, una correspondiente a comprensión lectora, y la otra orientada al manejo de matemáticas. Dado que el modelo planteado busca explicar "el rendimiento escolar" en un sentido amplio, se decidió considerar la existencia de una única variable dependiente, que vendría a ser el promedio de ambos resultados.

\section{RESULTADOS}

Tabla $\mathrm{N}^{\circ} 1$

Variables explicativas

\begin{tabular}{|l|l|l|l|}
\hline Nombre & Descripción & Tipo & Efecto esperado \\
\hline AÑOS & $\begin{array}{l}\text { Promedio de años de } \\
\text { duración de estudios } \\
\text { primarios }\end{array}$ & $\begin{array}{l}\text { Variable } \\
\text { interna } \\
\text { (generada por } \\
\text { el sistema } \\
\text { educativo) }\end{array}$ & $\begin{array}{l}\text { A mayor cantidad de años en la } \\
\text { educación primaria, mayor tiempo de } \\
\text { formación básica (pero no es clara la } \\
\text { relación con esta variable, pues a su } \\
\text { vez se asume que la duración de los } \\
\text { estudios primarios crece por repitencia, } \\
\text { es decir, el efecto contrario. }\end{array}$ \\
\hline
\end{tabular}




\begin{tabular}{|c|c|c|c|}
\hline DESERCIÓN & $\begin{array}{l}\text { Tasa de deserción } \\
\text { escolar en primaria } \\
\text { (\% de alumnos } \\
\text { matriculados) }\end{array}$ & $\begin{array}{l}\text { Variable } \\
\text { interna } \\
\text { (generada por } \\
\text { el sistema } \\
\text { educativo) }\end{array}$ & $\begin{array}{l}\text { Efecto negativo: a menor deserción, } \\
\text { mayor permanencia en las escuelas, por } \\
\text { tanto, mayor posibilidad de una mejor } \\
\text { formación del estudiante. }\end{array}$ \\
\hline COMPENSACIONES & $\begin{array}{l}\text { Gasto en } \\
\text { compensaciones } \\
\text { económicas a } \\
\text { maestros en } \\
\text { instituciones públicas } \\
\text { (\% de gasto público } \\
\text { en educación) }\end{array}$ & $\begin{array}{l}\text { Variable } \\
\text { interna } \\
\text { (generada por } \\
\text { el sistema } \\
\text { educativo) }\end{array}$ & $\begin{array}{l}\text { A mayor nivel de compensaciones } \\
\text { económicas a maestros debería notarse } \\
\text { una mejor predisposición a la } \\
\text { enseñanza, por tanto deberían tenerse } \\
\text { mejores resultados en la formación } \\
\text { escolar. }\end{array}$ \\
\hline PBI/PERCAPITA & $\begin{array}{l}\text { Producto Bruto } \\
\text { Interno Per Cápita } \\
\text { (en miles de US\$ } \\
\text { constantes del } 2000 \text { ) }\end{array}$ & $\begin{array}{l}\text { Variable } \\
\text { externa (del } \\
\text { entorno, } \\
\text { condiciones } \\
\text { macroeconómi } \\
\text { cas, bienestar) }\end{array}$ & $\begin{array}{l}\text { Efecto positivo: a mayor nivel de renta } \\
\text { y riqueza familiar, mejores } \\
\text { posibilidades para contar con un } \\
\text { entorno propicio a un mejor } \\
\text { rendimiento escolar. }\end{array}$ \\
\hline INTERNET & $\begin{array}{l}\text { Número de usuarios } \\
\text { de Internet (por cada } \\
100 \text { habitantes) }\end{array}$ & $\begin{array}{l}\text { Variable } \\
\text { externa (del } \\
\text { entorno, } \\
\text { condiciones } \\
\text { macroeconómi } \\
\text { cas, bienestar) } \\
\end{array}$ & $\begin{array}{l}\text { Efecto positivo: a mayor nivel de } \\
\text { bienestar (contar con servicios de } \\
\text { comunicación y TI), mejores } \\
\text { posibilidades para contar con un } \\
\text { entorno propicio a un mejor } \\
\text { rendimiento escolar. }\end{array}$ \\
\hline REPITENTES & $\begin{array}{l}\text { Porcentaje de } \\
\text { repitentes en } \\
\text { educación primaria }\end{array}$ & $\begin{array}{l}\text { Variable } \\
\text { interna } \\
\text { (generada por } \\
\text { el sistema } \\
\text { educativo) }\end{array}$ & $\begin{array}{l}\text { Efecto negativo: a menor cantidad de } \\
\text { repitentes, mayor capacidad de las } \\
\text { escuelas para promover un mejor } \\
\text { rendimiento de sus alumnos (por el } \\
\text { efecto analizado de }\end{array}$ \\
\hline COMPUTADORAS & $\begin{array}{l}\text { Computadoras } \\
\text { personales (por cada } \\
100 \text { habitantes) }\end{array}$ & $\begin{array}{l}\text { Variable } \\
\text { externa (del } \\
\text { entorno, } \\
\text { condiciones } \\
\text { macroeconómi } \\
\text { cas, bienestar) }\end{array}$ & $\begin{array}{l}\text { Efecto positivo: a mayor nivel de } \\
\text { bienestar (contar con servicios de } \\
\text { comunicación y TI), mejores } \\
\text { posibilidades para contar con un } \\
\text { entorno propicio a un mejor } \\
\text { rendimiento escolar. }\end{array}$ \\
\hline GASTO PRIMARIA & $\begin{array}{l}\text { Gasto público en } \\
\text { educación primaria } \\
\text { (\% de gasto público } \\
\text { en educación) }\end{array}$ & $\begin{array}{l}\text { Variable } \\
\text { interna } \\
\text { (generada por } \\
\text { el sistema } \\
\text { educativo) }\end{array}$ & $\begin{array}{l}\text { Efecto positivo: a mayor proporción } \\
\text { del gasto en educación orientado a la } \\
\text { formación básica (primaria) respecto a } \\
\text { la totalidad del gasto en educación, } \\
\text { mejores resultados se obtendrán en este } \\
\text { nivel. }\end{array}$ \\
\hline $\begin{array}{l}\text { GASTOEDUCACION } \\
\text { PBI }\end{array}$ & $\begin{array}{l}\text { Gasto público en } \\
\text { educación ( } \% \text { del } \\
\text { Producto Bruto } \\
\text { Interno) }\end{array}$ & $\begin{array}{l}\text { Variable } \\
\text { interna } \\
\text { (generada por } \\
\text { el sistema } \\
\text { educativo) }\end{array}$ & $\begin{array}{l}\text { Efecto positivo: a mayor proporción } \\
\text { del gasto orientado a la educación en } \\
\text { general, mejores resultados se } \\
\text { obtendran en el rendimiento escolar. }\end{array}$ \\
\hline $\begin{array}{l}\text { RATIOALUMNOMA } \\
\text { ESTRO }\end{array}$ & $\begin{array}{l}\text { Ratio } \\
\text { alumnos/maestros en } \\
\text { educación primaria }\end{array}$ & $\begin{array}{l}\text { Variable } \\
\text { interna } \\
\text { (generada por } \\
\text { el sistema } \\
\text { educativo) }\end{array}$ & $\begin{array}{l}\text { Efecto negativo: a menor el ratio } \\
\text { alumnos/maestros, se asume mejores } \\
\text { condiciones en el aula para desarrollar } \\
\text { la labor académica. }\end{array}$ \\
\hline
\end{tabular}

Finalmente, se tomaron en cuenta los datos de 23 países de todo el mundo, representativos de distintos niveles de desarrollo económico y social. 
Tabla $\mathrm{N}^{\circ} 2$

Correlaciones

\begin{tabular}{|c|c|c|c|c|c|c|c|c|c|c|c|c|}
\hline & & PISA & $\mathrm{Al} /$ & B 2/ & $\mathrm{C} 3 /$ & D 4/ & E 5/ & F 6/ & $\mathrm{G} 7 /$ & $\mathrm{H} 8$ / & $19 /$ & $\mathrm{J} 10 /$ \\
\hline \multirow[t]{3}{*}{ PISA } & $\begin{array}{l}\text { Corr } \\
\text { elaci } \\
\text { on de } \\
\text { Pears } \\
\text { on }\end{array}$ & 1 & 0,234 & $-0,766^{* *}$ & \begin{tabular}{|c|}
$-0,383$ \\
\end{tabular} & $0,581^{* *}$ & $0,813^{* *}$ & $-0,644^{* *}$ & $0,733^{* \prime}$ & $-0,626^{* 7}$ & $0,576^{\prime \prime *}$ & $-0,514^{4}$ \\
\hline & $\begin{array}{l}\text { Sig. } \\
\text { (bilat } \\
\text { eral) }\end{array}$ & & 0,282 & 0,000 & 0,071 & 0,004 & 0,000 & 0,004 & 0,000 & 0,003 & 0,004 & 0,014 \\
\hline & $\mathrm{N}$ & 23 & 23 & 23 & 23 & 23 & 23 & 18 & 23 & 20 & 23 & 22 \\
\hline
\end{tabular}

**. La corrélación es significatıva al nivel 0,01 (bilateral).

*. La correlación es significante al nivel 0,05 (bilateral).

1/ Años; 2/ Deserción; 3/ Compensaciones, 4/ PBI perca pita ; 5/ INTERNET; 6/ Repitentes; 7 / Computadoras; 8/ Gasto primaria; 9/ Gasto educación PBI; 10/ Ratio alumno/maestro

La variable INTERNET es la que presentó los mejores niveles de ajuste en el modelo de regresión. La regresión muestra un coeficiente de determinación $\mathrm{R}^{2}$ de 0.662, con un coeficiente de correlación de Pearson bastante elevado, de 0.813 y en sentido positivo (corroborando el efecto inicialmente esperado). El coeficiente determinación muestra que un $66.2 \%$ de la variación total de la variable dependiente (PISA) se explica por la variación de la variable independiente (INTERNET).

El ratio $\mathrm{F}$ tiene un valor de 41.067 , lo que indica cuánto ha mejorado el modelo de predicción en relación al nivel de precisión del modelo. Esta es la variabilidad explicada por el modelo, en comparación con la variabilidad promedio no explicada por el mismo modelo, y supera la prueba con una significancia del $1 \%$. La prueba $\mathrm{F}$ de significancia demuestra que hay suficiente evidencia de que la variable INTERNET afecta a los resultados manifestados en la variable PISA.

\section{Tabla $N^{\circ} 3$}

\section{Coeficientes de regresión}

\begin{tabular}{|c|c|c|c|c|c|c|c|c|c|c|c|c|}
\hline \multirow[b]{2}{*}{ Modelo } & \multicolumn{2}{|c|}{$\begin{array}{l}\text { Coeficientes no } \\
\text { estandarizados }\end{array}$} & \multirow{2}{*}{\begin{tabular}{|c|}
$\begin{array}{c}\text { Coeficientes } \\
\text { tipificados }\end{array}$ \\
Beta \\
\end{tabular}} & \multirow[b]{2}{*}{$\mathrm{t}$} & \multirow[b]{2}{*}{ Sig. } & \multicolumn{2}{|c|}{$\begin{array}{l}\text { Intervalo de } \\
\text { confianza de } \\
95.0 \% \text { para B }\end{array}$} & \multicolumn{3}{|c|}{ Correlaciones } & \multicolumn{2}{|c|}{$\begin{array}{c}\text { Estadísticos de } \\
\text { colinealidad }\end{array}$} \\
\hline & B & $\begin{array}{l}\text { Error } \\
\text { típ. }\end{array}$ & & & & \begin{tabular}{|l|} 
Limite \\
inferior
\end{tabular} & $\begin{array}{l}\text { Limite } \\
\text { superior }\end{array}$ & $\begin{array}{l}\text { Orden } \\
\text { cero }\end{array}$ & Parcial & Semiparcial & Tolerancia & FIV \\
\hline 1 (Constante) & 372,348 & 15,562 & & 23,927 &, 000 & 339,985 & 404,710 & & & & & \\
\hline INTERNET & 2,252 & 0,351 & 0,813 & 6,408 &, 000 & 1,521 & 2,983 & 0,813 & 0,813 & 0,813 & 1,000 & 1,000 \\
\hline
\end{tabular}

Ambos coeficientes muestran un estadístico $\mathrm{t}$ que nos lleva a rechazar las hipótesis nulas, con un nivel de significancia del 1\%. La inferencia sobre la pendiente nos lleva a aceptar que hay una relación entre PISA e INTERNET. La 
constante muestra un intervalo de confianza al 95\% que va de 339.985 a 404.710. La pendiente muestra un intervalo de confianza al $95 \%$ que va de 1.521 a 2.983. Es claro que el intervalo de confianza no incluye el valor cero (0).

Como vemos, el modelo de regresión lineal es el que mejor representa los datos de la dispersión de puntos. No se rompe el supuesto de linealidad, pues se observa que la relación entre las variables PISA e INTERNET es básicamente lineal. No se violan los supuestos principales para la regresión. Encontramos niveles razonables de linealidad, independencia de errores, normalidad del error y homoscedasticidad.

\section{DISCUSIÓN}

Queda claro que la variable independiente INTERNET (número de usuarios de Internet en el país por cada 100 habitantes) no es en sí misma un condicionante de los resultados de las pruebas de medición de la calidad educativa PISA (en este caso, el promedio de las pruebas PISA para comprensión lectora y matemáticas). Sin embargo, se establece una importante relación entre lo que significa la penetración de Internet en los hogares (como medida de progreso material, desarrollo en infraestructura de comunicaciones y tecnologías de información) (Meneses et al., 2005) y los resultados encontrados en las evaluaciones de rendimiento académico en el nivel primario en los mismos países.

En general, si bien no se ha analizado al detalle las posibles regresiones lineales adicionales que pueden realizarse con las otras variables independientes encontradas, queda claro que son las variables que han sido calificadas como externas o del entorno, las que tienen una mayor injerencia en la determinación de la variable dependiente. En otras palabras, son las variables externas las que tienen un mayor poder explicativo sobre los resultados, tal como se señaló como premisa en estudios previos.

Es importante señalar que esta conclusión se está dando en un contexto global, a diferencia de la mayoría de estudios previos, donde se analizaban las condiciones socioeconómicas y del entorno al interior de cada país o región. En este caso cada país analizado es tomado como un grupo homogéneo, que se manifiesta dentro de un contexto único, con determinados niveles de desarrollo, de bienestar, de condiciones socioeconómicas y de infraestructura.

Naturalmente, al trabajar sobre promedios nacionales y compararlos entre sí, estamos obviando las "colas" que se presentan en cada realidad concreta de cada país. Es innegable que dentro de países en desarrollo (con menores niveles de desarrollo y condiciones socioeconómicas), podemos encontrar en ciertos estratos suficientes condiciones del entorno que puedan generar resultados elevados en las pruebas de rendimiento escolar. Igual en sentido inverso, pues en países desarrollados, la educación pública en zonas de menor desarrollo relativo ( $y$ en algunos casos simplemente en urbes con condiciones de bienestar no óptimas) puede dar como resultado niveles bajos en las pruebas de rendimiento escolar. 


\section{CONCLUSIÓN}

Esta investigación ha simplificado a nivel global un modelo que buscaba probar que, las premisas que se generaron hace varias décadas sobre la importancia de factores no directamente ligados al sistema educativo, aún siguen vigentes, y que son aquellos elementos del entorno, de las condiciones macroeconómicas y de bienestar, los que ejercen una mayor influencia sobre los resultados de las pruebas de rendimiento escolar.

\section{REFERENCIAS BIBLIOGRÁFICAS}

$>$ Agüero, J. \& Cueto, S. (2004). Dime con quién estudias y te diré cómo rindes: Peer-effects como determinantes del rendimiento escolar. Economía y Sociedad 54. Lima: CIES.

- Aitken, M. \& Longford, N. (1986). Statistical modeling issues in school effectiveness studies. Journal of the Royal Statistical Society, Series A, 149 (1), $1-43$.

> Carlson, B. (2000). ¿Qué nos enseñan las escuelas sobre la educación de los niños pobres en Chile? Revista de la CEPAL 72. Santiago de Chile: CEPAL.

$>$ Coleman, J. et al. (1966). Equality of Educational Opportunity. Washington, D.C.: US. Government Printing Office.

> Creemers, B. (1992). School effectiveness, effective instruction and school improvement in the Netherlands. School effectiviness. Research, policy and practice. Nueva York: Casell.

> Espínola, V. \& Martínez, R. (1996). Importancia relativa de algunos factores del rendimiento educativo: consulta a expertos. Santiago de Chile: CEPAL.

$>$ Himmel, E. et al. (1984). Análisis de la influencia de factores alterables del proceso educativo sobre la efectividad escolar. Santiago de Chile: Universidad Católica de Chile.

> Jencks, C. et al. (1971). Inequality. Londres: Allen Lane.

> Lockheed, M. \& Bruns, B. (1990). School effects on achievement in secondary mathematics and Portuguese in Brazil. Washington: Banco Mundial.

- Mella, O. \& Ortiz, I. (1999). Rendimiento escolar. Influencias diferenciales de factores externos e internos. Revista Latinoamericana de Estudios Educativos (México), vol. XXIX (1), 69 - 92.

$>$ Meneses, J. et al. (2005). La escuela eficaz en la sociedad red: el uso de Internet y la generación de capital social en la relación de la escuela con la comunidad y el territorio. Revista Electrónica Iberoamericana sobre Calidad, Eficacia y Cambio en Educación. Vol. 3 (1). http://www.ice.deusto.es/rinace/reice/vol3n1_e/Menesesetal.pdf

- Mizala, A. et al. (1999). Factores que inciden en el rendimiento escolar en Bolivia. Documentos de Trabajo 61. Centro de Economía Aplicada Departamento de Ingeniería Industrial, Universidad de Chile.

> Mortimore, P. et al. (1988). School Matters: The Junior Years. Salisbury: Open Books.

> Saavedra, J. et al. (1997). Financiamiento de la educación en el Perú. Documento de Trabajo 24. Lima: Grade.

Fuente de estadísticas: http://data.worldbank.org/indicator

Recibido: 02/11/2015

Aceptado para publicación:

$17 / 11 / 2015$ 